\title{
ÉTUDE THROM BÉLASTOGRAPHIQUE DE LA COAGULATION DU LAIT PAR LA PRÉSURE: ACTION DE LA TEMPÉRATURE ET DE LA CONCENTRATION EN CALCIUM
}

\author{
par \\ B. TARODO DE LA FUENTE et R. FRENTZ \\ (Ecole de Laiterie de Nancy. Directeur : A. Veillet)
}

La technique thrombélastographique de Hartert [1], [2], [3], [4] adaptée au lait, pourra peut-être un jour rendre des services importants à l'industrie fromagère, car elle permet d'observer, de concrétiser et de mesurer un phénomène dont l'interprétation pratique est généralement laissée aux sens de fromagers expérimentés, mais faillibles.

$\mathrm{Ne}$ permet-elle pas, entre autres, de conserver l'image exacte d'une coagulation particulière, de la reproduire fidèlement si l'on prend soin de se placer dans des conditions physiques et chimiques identiques, alors que d'autres appareils ne permettent que la mesure du temps de prise [5].

Certes, on peut lui reprocher de ne travailler que sur de très petites quántités de lait, donc de ne pas reproduire les conditions d'une fabrication industrielle, mais cela n'est pas forcément un inconvénient majeur, car il est fôrt probable qu'il existe une corrélation entre le phénomène tel qu'il se déroule dans une cuve industrielle et son développement dans la petite cuve du thrombélastographe. Une transposition de l'un à l'autre doit done toujours être possible, mais avant de pouvoir en juger, il est indispensable de connaître en détail le comportement thrombélastographique d'un lait en voie de coagulation en faisant varier un à un les principaux facteurs qui peuvent l'influencer.

Dans une première étape, nous nous sommes done attachés à étudier l'influence de la température d'emprésurage sur le temps de prise thrombélastographique de différents laits, puis nous avons considéré l'action de la teneur en calcium.

\section{I. - Etude de l'influence de la température d'emprésurage}

\section{A) Signification des paramètres utilisés. \\ 1) Paramètre $r$.}

Exprimé en minutes et secondes, il mesure le temps écoulé depuis l'emprésurage jusqu'au moment où la largeur du tracé 
thrombélastographique est de $1 \mathrm{~mm}$. Il représente le "temps de prise thrombélastographique " et ne correspond pas exactement au temps de prise défini en pratique.

Pour avoir une idée du décalage existant entre ces temps, nous avons fait les essais suivants :

Un lait reconstitué à partir de poudre Spray est emprésuré dans un bécher de $300 \mathrm{ml}$. Une partie en est immédiatement prélevée et placée dans la cuve du thrombélastographe, lequel est mis en route et fournit $r$, la mesure étant faite à la plus grande sensibilité. Dans le bécher maintenu à la même température que la cuve, la floculation est appréciée au moyen d'un tube capillaire d'un millimètre de diamètre dont l'extrémité, plongeant dans le lait, est effilée de façon à réduire d'au moins 20 fois son diamètre interne. Le tube est coudé trois fois à angle droit (fig. 1) de façon à créer un siphon qui, une fois amorcé par aspiration, permet l'écoulement lent du lait jusqu'à l'instant précis de la floculation dont le débit peut être ainsi chronométré avec rigueur. Les temps obtenus sont indiqués dans le tableau I.

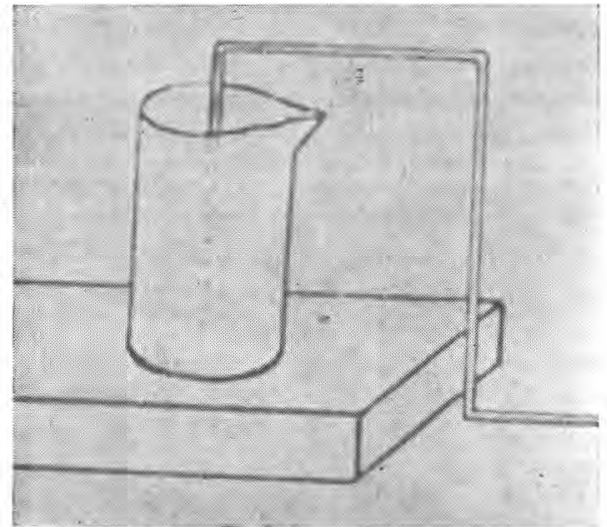

FIG. 1. - Dispositif hydrodynamique pour la détermination du début de la coagulation d'un lait emprésuré.

TABLEAU I

\begin{tabular}{|c|c|c|c|c|c|}
\hline $\begin{array}{c}\text { Temps } \\
\text { hydrodynamique } \\
r \\
\text { Ecarts }\end{array}$ & $\begin{array}{r}7 \mathrm{mn} 17 \mathrm{~s} \\
7-30- \\
13-\end{array}$ & $\begin{array}{r}7 \mathrm{mn} 01 \mathrm{~s} \\
7-36- \\
35-\end{array}$ & $\begin{array}{r}6 \mathrm{mn} 54 \mathrm{~s} \\
7-21- \\
27-\end{array}$ & $\begin{array}{r}6 \mathrm{mn} 43 \mathrm{~s} \\
7-18- \\
35-\end{array}$ & $\begin{array}{r}6 \mathrm{mn} 50 \mathrm{~s} \\
7-18- \\
28-\end{array}$ \\
\hline
\end{tabular}

Ces écarts, qui sont de l'ordre d'une demi minute, ne sont qu'indicatifs et valables seulement pour les conditions expérimentales choisies. Ils seront plus faibles pour des coagulations plus rapides et plus importants pour des coagulations plus progressives. 
Ils ne doivent pas être interprétés comme un manque de sensibilité de l'appareil par rapport à la méthode d'écoulement, mais simplement comme la conséquence du choix arbitraire, mais commode, d'une largeur de $1 \mathrm{~mm}$ du tracé. En effet, si on examine ce tracé à la loupe, on s'aperçoit que les premiers signes de variation des propriétés mécaniques du contenu de la cuve sont perceptibles au moins 1 minute avant le point de mesure de $r$, donc aussi avant leur mise en évidence par le système d'écoulement. Le thrombélastographe est done, en fait, plus sensible que le système de détection hydrodynamique utilisé comparativement.

\section{2) Paramètre $k$.}

Nous rappelons que c'est le temps mis par le coagulum pour acquérir certaines propriétés mécaniques définies par une largeur de $20 \mathrm{~mm}$ du thrombélastogramme. En effet, étant donné le principe de fonctionnement de l'appareil, à chaque caractéristique mécanique du caillé correspond un écartement particulier des bords du thrombélastogramme et on peut admettre réciproquement qu'un écartement donné représente à l'instant $t$ un état physique particulier du caillé. D'après les nombreuses constatations que nous avons pu faire, l'écart obtenu varie directement avec la fermeté du coagulum : plus celui-ci est ferme, plus l'écartement des deux branches de la courbe est important.

\section{3) Paramètre Am.}

Il mesure l'écartement maximum des deux branches du thrombélastogramme. C'est donc une représentation des propriétés mécaniques maxima du caillé.

\section{B) Appareillage utilisé.}

\section{1) Description.}

Tous les essais ont été faits avec un thrombélastographe Hellige (1) (fig. 2), dont deux des unités de mesure ont été dotées d'une sensibilité nettement supérieure à celle de l'appareil commercial. En ce qui concerne les problèmes étudiés ici, nous avons toujours utilisé la sensibilité maximum de façon à obtenir les plus grandes différences possibles dès que l'un des facteurs a été modifié.

Les caractéristiques thermiques de l'appareil ont également été transformées. Alors que le type courant est conçu pour travailler uniquement à $37^{\circ}$, le modèle dont nous disposons permet de faire varier aisément la température de 0 à $50^{\circ}$ et même au-delà. Pour cela, le thermomètre de contact d'origine a été remplacé par un thermomètre courant permettant une lecture au $1 / 10^{\mathrm{e}}$ de degré.

(1) Hellige-France, 80, route de Bischwiller (67), Strasbourg-Schiltigheim. 
La plaque de fondation chargée d'assurer et de maintenir dans les cuves la température choisie a été dotée d'une canalisation interne permettant la circulation d'un courant de liquide, d'eau par exemple. La température de l'eau, donc celle des cuves du thrombélastographe, est réglée par un thermostat à circulation Haake (fig. 2) fourni avec l'appareil. Ce thermostat est un bain-marie

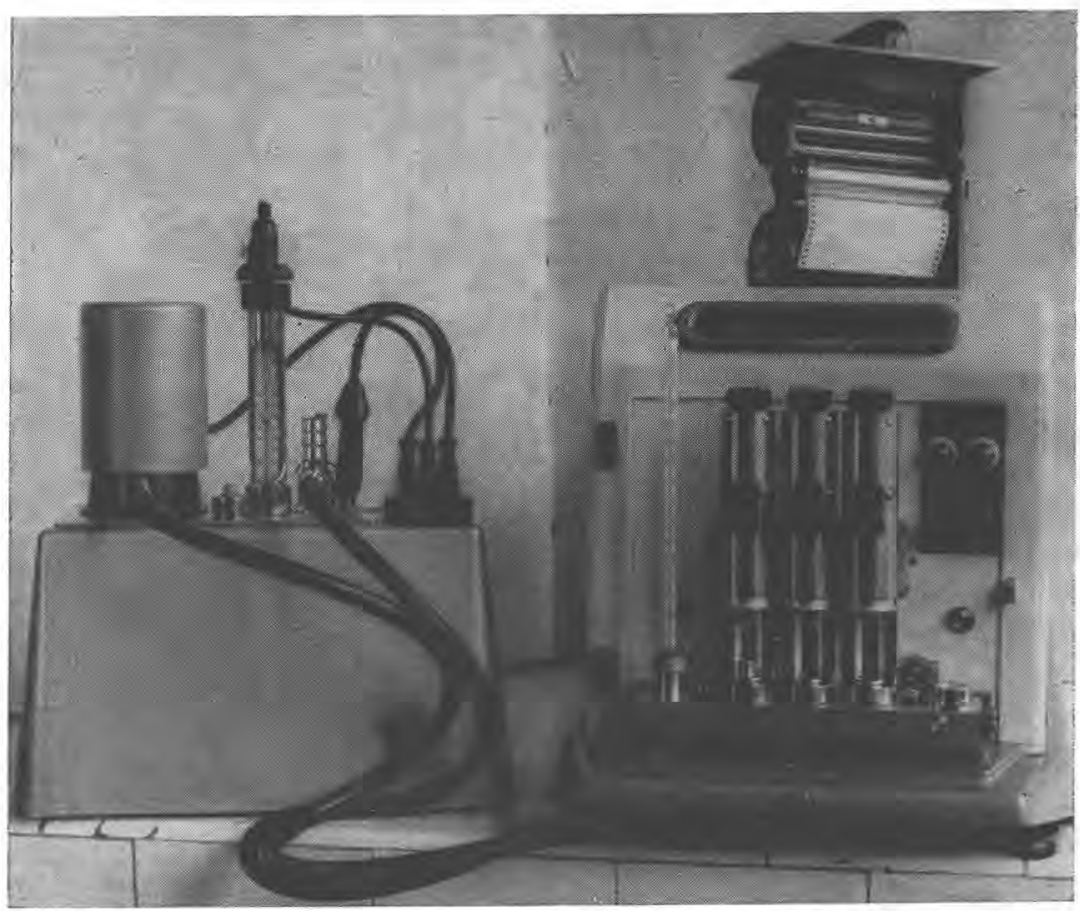

FIG. 2. - Thrombélastographe Hellige avec thermostat à circulation Haake.

clos comportant un système de chauffage par thermoplongeur et un système de refroidissement par circulation de liquide réfrigéré, ce dernier permettant de travailler à une température inférieure à la température ambiante. Une pompe immergée connectée à la plaque de fondation du thrombélastographe assure une circulation de liquide à l'intérieur de celle-ci. La température du liquide est réglée par un thermomètre à contact type Vertex. Au point de vue amplitude et variation de température, le système de chauffage et de régulation est excellent : la température se règle au $1 / 10^{\mathrm{e}}$ de degré et comme la pompe est dotée de deux vitesses de rotation, on peut passer de la température ambiante à $50^{\circ} \mathrm{C}$ en moins de 15 minutes. 
Il faut cependant remarquer que la plaque de fondation n'habille pas entièrement les cuves de mesure, ce qui peut entraîner une différence de température entre elles et le thermomètre de contrôle. Cette différence est mesurable par un deuxième thermomètre placé dans l'une des cuves voisines. Au cours des essais effectués, nous n'avons jamais constaté de variation de température dans les cuves tant que les variations de la température ambiante ne dépassaient pas 3 ou $4^{\circ} \mathrm{C}$. Dans le cas où de telles variations deviendraient gênantes pour la rigueur de l'expérimentation, il serait aisé d'y remédier en plaçant l'appareil dans une enceinte à température constante.

\section{2) Reproductibilité.}

C'est une exigence indispensable à laquelle l'appareil doit satisfaire. Les premiers essais faits par l'un de nous avaient déjà montré que les einq thrombodynamogrammes obtenus dans la même journée avec des laits de vache crus écrémés étaient pratiquement identiques [4]. Depuis, nous nous sommes efforcés de préciser la variation que l'on peut trouver entre deux essais semblables.

Le substrat utilisé est du lait en poudre Spray reconstitué à 10 p. 100 et additionné de $3 \mathrm{ml}$ de chlorure de calcium à 20 p. 100 pour $100 \mathrm{ml}$ de lait. Cette quantité de calcium et la dose de présure ont été fixées arbitrairement en tenant compte simplement du temps de prise choisi voisin de 7 minutes. Tous les essais sont faits à une température de coagulation identique à la température d'emprésurage et égale à $37^{\circ} \mathrm{C}$. La présure employée est de la présure liquide Hauser, de force 10000 , mais préalablement diluée 10 fois avec de l'eau distillée. L'emprésurage est fait à raison de $1 \mathrm{ml}$ de présure diluée pour $100 \mathrm{ml}$ de lait.

Les résultats obtenus figurent au tableau II.

TABLEAU II

\begin{tabular}{|c|c|c|c|c|c|}
\hline $\begin{array}{c}\text { Numéro } \\
\text { de } \\
\text { l'essai }\end{array}$ & $r$ & $k$ & $\begin{array}{c}\text { Numéro } \\
\text { de } \\
\text { l'essai }\end{array}$ & $r$ & $k$ \\
\hline $\begin{array}{l}1 \\
2 \\
3 \\
4 \\
5 \\
6\end{array}$ & $\begin{array}{l}7 \mathrm{mn} 30 \mathrm{~s} \\
7-24= \\
7-30- \\
7-30= \\
7=36= \\
7-33-\end{array}$ & $\begin{array}{l}6 \mathrm{mn} 30 \mathrm{~s} \\
6-39- \\
6-30- \\
6-30- \\
6-30- \\
6-24-\end{array}$ & $\begin{array}{r}7 \\
8 \\
9 \\
10 \\
11 \\
12 \\
13\end{array}$ & $\begin{array}{l}7 \mathrm{mn} 36 \mathrm{~s} \\
7-21- \\
7-21- \\
7-30- \\
7-33- \\
7-36- \\
7-33-\end{array}$ & $\begin{array}{l}6 \mathrm{mn} 30 \mathrm{~s} \\
6-24- \\
6-24- \\
6-24- \\
6-30- \\
6-30- \\
6-24-\end{array}$ \\
\hline
\end{tabular}


Pour le paramètre $r$ la moyenne est égale à $7 \mathrm{mn} 30 \mathrm{~s}$ et l'écarttype à 5 secondes.

Pour $k$, la moyenne est égale à $6 \mathrm{mn} 28 \mathrm{~s}$ et l'écart-type à 4 secondes.

l. La reproductibilité de la méthode est donc satisfaisante.

\section{C) Action de la température.}

1) Cas d'un lait reconstitué à partir de poudre.

a) Substrat utilisé.

Le lait est reconstitué à partir de poudre Spray (France-Lait) à raison de $10 \mathrm{~g}$ de poudre pour un volume final de $100 \mathrm{ml}$. Après reconstitution, la dissolution est contrôlée par filtration. Les caractéristiques chimiques de ce lait sont les suivantes :

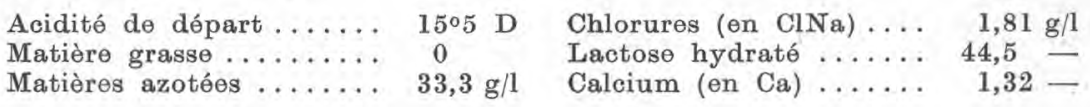

b) Mode opératoire.

Le lait est préparé au moment de l'emploi et une partie aliquote de $100 \mathrm{ml}$ est placée dans un bécher maintenu dans un bain-marie à la température de coagulation désirée. Il est ensuite additionné de $0,3 \mathrm{ml}$ d'une solution de chlorure de calcium à 20 p. 100 et emprésuré par $1 \mathrm{ml}$ de présure diluée au $1 / 10^{\mathrm{e}}$ avec de l'eau distillée à partir d'une présure Hauser de force 10000 . Immédiatement après emprésurage et agitation pendànt 20 secondes, on pipette $0,35 \mathrm{ml}$ de lait que l'on porte dans la cuve déjà en place sur l'appareil. Le cylindre de mesure est alors plongé dans le lait que l'on recouvre d'huile de paraffine pour éviter l'évaporation et la formation d'une peau en surface. Le système enregistreur est mis en route 2 ou 3 minutes exactement après emprésurage, suivant le temps de prise présumé.

Notre but étant uniquement d'étudier l'évolution du temps de prise et du début de la coagulation en fonction de la température, nous avons arrêté chaque essai au bout d'un temps fixé à 2 heures.

c) Résultats et discussion.

Nous avons fait varier la température de 2 en 2 , de 29 à $50^{\circ} \mathrm{C}$, l'étude du phénomène au-delà de ces limites devant faire l'objet d'essais ultérieurs. Les valeurs obtenues sont consignées dans le tableau III et ont permis d'établir les courbes de la figure 3. De 29 à $39^{\circ} \mathrm{C}$, on constate une diminution rapide du temps de prise au fur et à mesure qu'augmente la température. Au-delà de $39^{\circ} \mathrm{C}$, la diminution de $r$ se poursuit, mais de façon atténuée, pour atteindre un minimum vers $45^{\circ} \mathrm{C}$. Puis, le temps de prise s'allonge, modérément d'abord, de façon accélérée à partir de $50^{\circ} \mathrm{C}$. 
TABLEAU III

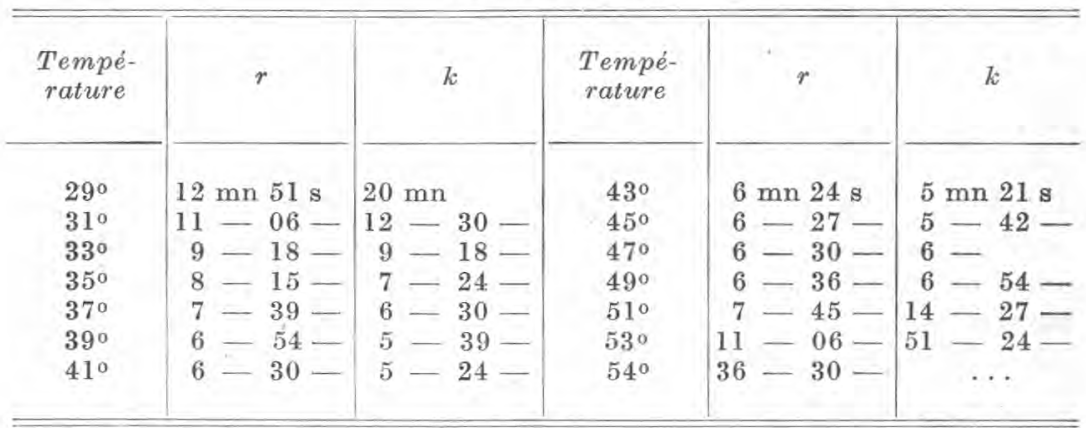

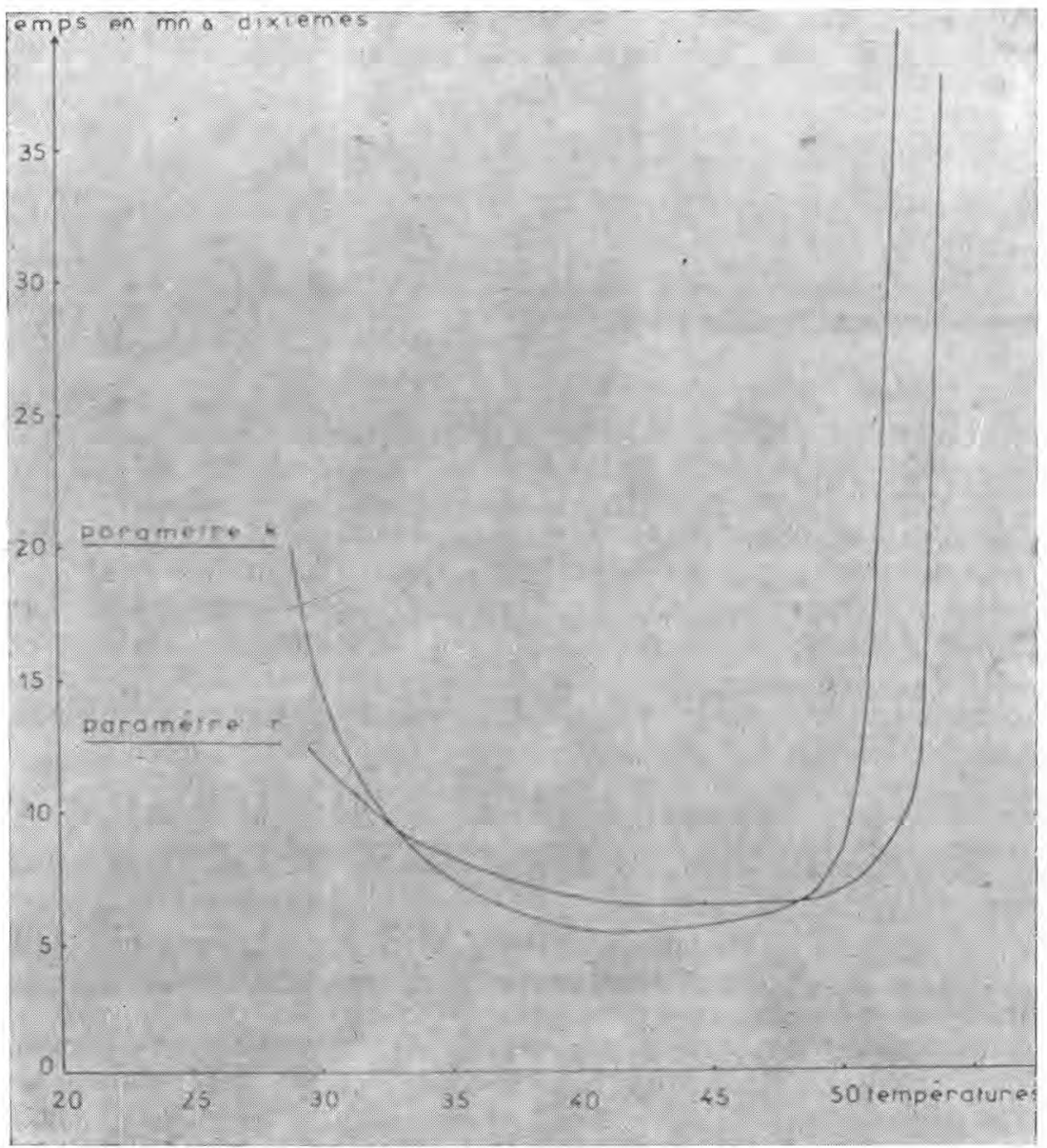

Fig. 3. - Courbes des variations de $r$ et de $k$ pour un lait reconstitué, en fonction de la température. 
Les variations des propriétés mécaniques du caillé représentées par $k$ vont dans le même sens et sont encore plus accusées. C'est ainsi qu'aux environs de $30^{\circ} \mathrm{C}, k$ est beaucoup plus élevé que $r$, alors qu'à $39^{\circ}$ la situation se trouve inversée. Comme $k$ représente en quelque sorte, la vitesse avec laquelle le coagulum acquiert sa consistance, on peut dire que le caillé atteint un certain état physique d'autant plus vite qu'on se rapproche de $39^{\circ} \mathrm{C}$.

On notera qu'à $54^{\circ} \mathrm{C}$, la coagulation est extrêmement ralentie, presque nulle. L'observation du caillé dans le bécher témoin montre qu'à cette température, il ne se forme qu'un coagulum très friable, semblable à un caillé uniquement "lactique " en début de coagulation.

Cette température limite fera l'objet d'une étude détaillée ultérieure, qui tiendra compte de l'acidité et de la teneur en calcium du lait, ainsi que de la dose de présure.

2) Cas d'un lait cru complet (fig. 4).

a) Substrat utilisé.

Les essais sont faits sur du lait cru complet prélevé dans un petit mélange de 400 à 600 litres.

b) Mode opératoire.

Comme le temps nécessaire pour obtenir des résultats qui couvrent l'intervalle de 26 à $50^{\circ} \mathrm{C}$ est d'environ 8 heures, il est préférable de répartir le lait au départ dans des ballons de $200 \mathrm{ml}$ qui seront maintenus au-dessous de $10^{\circ} \mathrm{C}$ jusqu'à leur utilisation. D'autre part, le lait est utilisé tel quel, sans addition de chlorure de calcium, et l'emprésurage est fait de la même manière que pour le lait reconstitué. Compte tenu du nombre d'essais à réaliser, et sauf cas particulier, chaque expérience est arrêtée au bout de 24 minutes seulement. Ce délai est assez court, mais suffisant pour obtenir les valeurs de $r$ et de $k$.

\section{c) Résultats et discussion.}

Les résultats consignés dans le tableau IV sont illustrés par les courbes de la figure 5 .

Comme pour un lait reconstitué, $r$ et $k$ diminuent au fur et à mesure que la température augmente jusqu'à environ $39^{\circ}$. Cette diminution est beaucoup plus rapide pour le paramètre $k$, sans doute parce que le caractère "fermeté du caillé " augmente beaucoup plus rapidement pour un lait cru que pour un lait reconstitué. On constate ensuite la présence d'un caillé de coagulation entre 39 et $45^{\circ} \mathrm{C}$. Au-dessus de $45^{\circ}$, les paramètres $r$ et $k$ augmentent rapidement et le coagulum obtenu devient de plus en plus mou. 


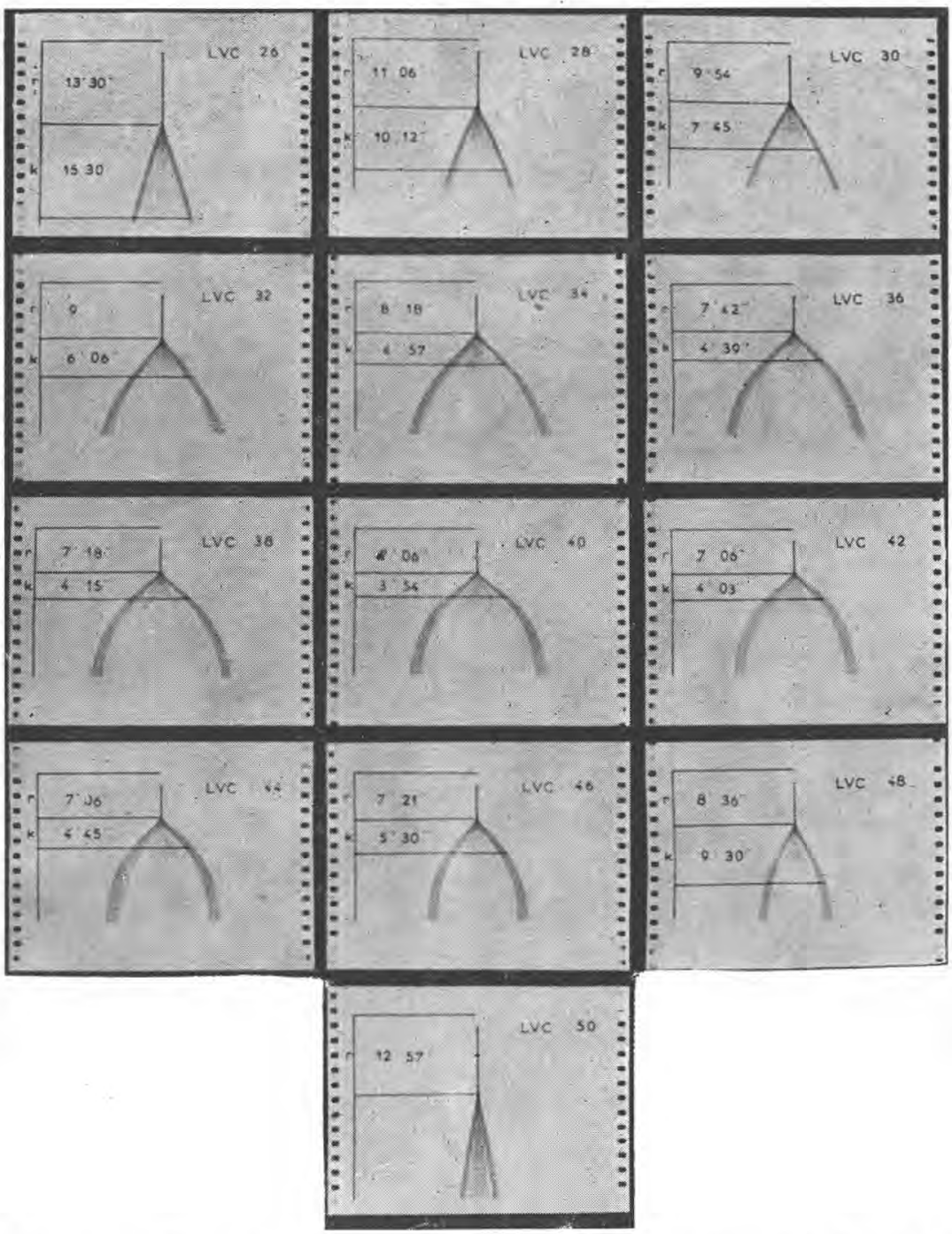

FIG. 4. - Thrombodynamogrammes partiels d'un même lait de vache complet, eru, à différentes températures d'emprésurage.

Au-dessus de $50^{\circ} \mathrm{C}$, la coagulation est très lente et dans les conditions de l'expérience, on ne peut plus parler de caillé. Nous avons effectué les mêmes séries d'essais sur différents laits. Toutes les courbes obtenues, tant pour $r$ que pour $k$, sont semblables. Seules diffèrent les valeurs absolues de $r$ et $k$, suivant la coagulation plus ou moins rapide du lait considéré sous l'action de la présure. C'est 


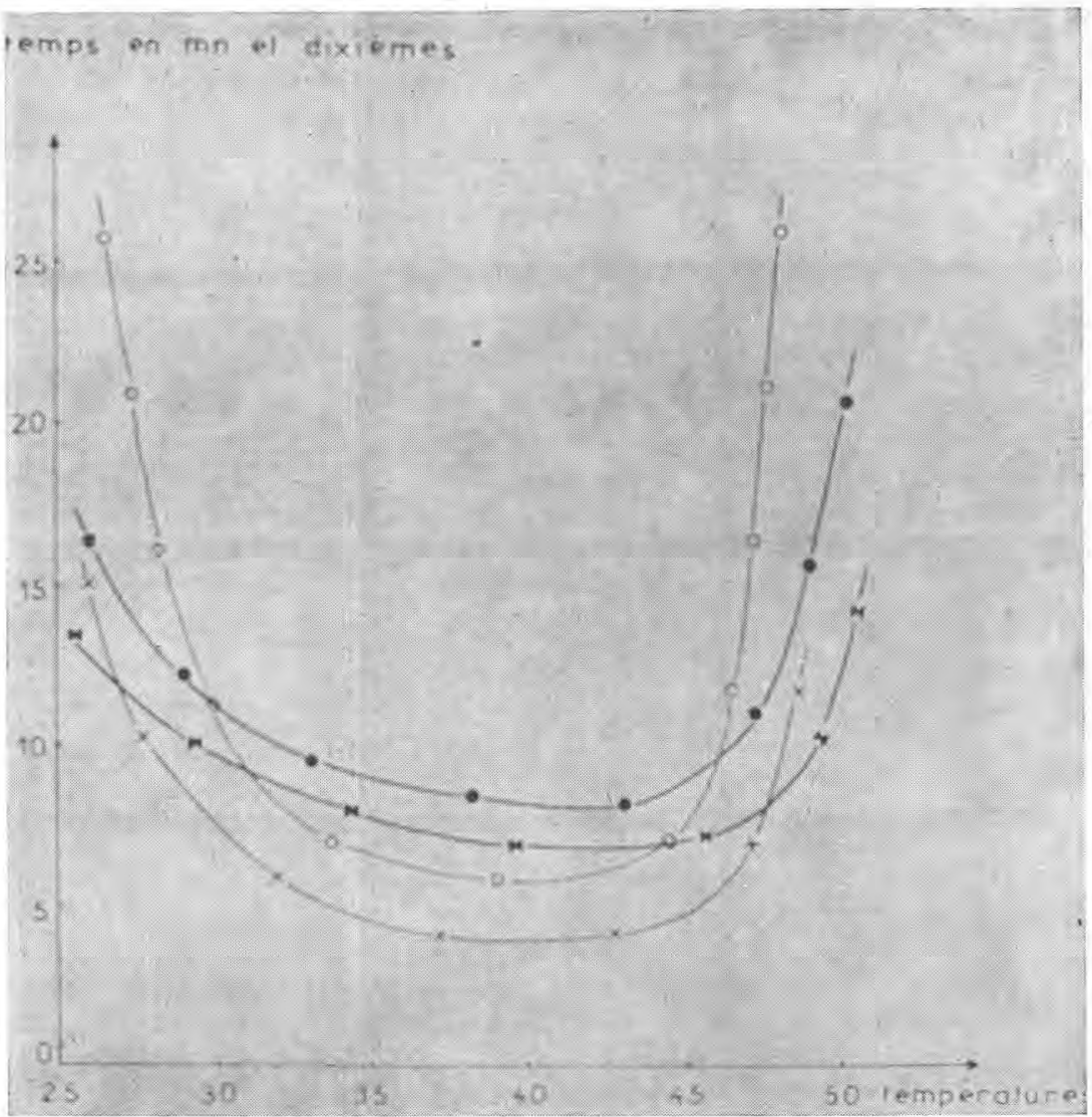

Fig. 5. - Courbes des variations de $r$ et de $k$ pour un lait de vachecomplet, cru et pour le même lait pasteurisé à $75^{\circ}$, en fonction de la température d'emprésurage.

$X=$ paramètre $k$ correspondant au lait cru

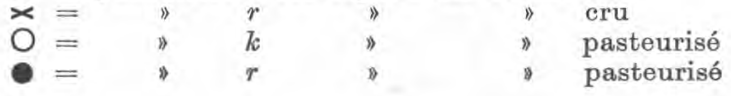

ainsi que pour le premier essai nous avions un temps de prise de $16 \mathrm{mn} 24 \mathrm{~s}$ à $28^{\circ} \mathrm{C}$, alors que pour le troisième essai nous avons observé un temps de $11 \mathrm{mn} 06 \mathrm{~s}$.

3) Cas d'un lait complet pasteurisé.

a) Substrat utilisé et mode opératoire.

Les essais sont faits sur le lait utilisé pour l'essai précédent mais pasteurisé à $75^{\circ} \mathrm{C}$ et conservé $24 \mathrm{~h}$ à $+4^{\circ} \mathrm{C}$. De même que 
pour le lait cru, il n'y a aucune addition de chlorure de calcium et l'emprésurage est fait d'une manière identique.

b) Résultats.

Le tableau IV donne les valeurs de $r$ et de $k$ dont les variations sont exprimées par les courbes correspondantes de la figure 5 . La variation de ces paramètres est analogue à celle constatée dans les essais précédents.

4) Comparaison des résultats sur lait cru et pasteurisé [6], [7] (tableau IV, fig. 5).

TABLEAU IV

\begin{tabular}{|c|c|c|c|c|c|}
\hline $\begin{array}{l}\text { Tempé- } \\
\text { rature }\end{array}$ & $\begin{array}{l}r \text { en lait } \\
\text { cru }\end{array}$ & $\begin{array}{l}r \text { en lait } \\
\text { pasteurisé }\end{array}$ & $\begin{array}{l}\text { Tempé- } \\
\text { rature }\end{array}$ & $\begin{array}{c}k \text { en lait } \\
\text { cru }\end{array}$ & $\begin{array}{l}k \text { en lait } \\
\text { pasteurisé }\end{array}$ \\
\hline $26^{\circ}$ & $13 \mathrm{mn} 30 \mathrm{~s}$ & $17 \mathrm{mn} 00 \mathrm{~s}$ & $26^{\circ}$ & $15 \mathrm{mn} 30 \mathrm{~s}$ & $29 \mathrm{mn} 15 \mathrm{~s}$ \\
\hline $28^{\circ}$ & $11-06-$ & $13-27-$ & $28^{\circ}$ & $10-12-$ & $17-48$ \\
\hline $30^{\circ}$ & $9-54-$ & $11-36-$ & $30^{\circ}$ & $7-45-$ & $11-30$ \\
\hline $32^{\circ}$ & $9-$ & $10-06-$ & $32^{\circ}$ & $6-06-$ & $8-24$ \\
\hline $34^{\circ}$ & $8-18-$ & $9-30-$ & $34^{\circ}$ & $4-57-$ & $7-30-$ \\
\hline $36^{\circ}$ & $7-42-$ & $8-57-$ & $36^{\circ}$ & $4-39-$ & $6-45-$ \\
\hline $38^{\circ}$ & $7-18-$ & $8-54-$ & $38^{\circ}$ & $4-15-$ & $6-$ \\
\hline $40^{\circ}$ & $7-06-$ & $8-24-$ & $40^{\circ}$ & $3-54-$ & $5-33-$ \\
\hline $42^{\circ}$ & $7-06-$ & $8-06-$ & $42^{\circ}$ & $4-03-$ & $6-12-$ \\
\hline $44^{\circ}$ & $7-06-$ & $8-24-$ & $44^{\circ}$ & $4-45-$ & $6-54$ \\
\hline $46^{\circ}$ & $7-21-$ & $9-33-$ & $46^{\circ}$ & $5-30-$ & $9-21-$ \\
\hline $48^{\circ}$ & $8-36-$ & $12-30-$ & $48^{\circ}$ & $9-30-$ & $26-12-$ \\
\hline $50^{\circ}$ & $12-57-$ & $\ldots$ & $50^{\circ}$ & $\ldots$ & $\ldots$ \\
\hline
\end{tabular}

Les essais ont été faits à $24 \mathrm{~h}$ d'intervalle pendant lesquelles le lait est stocké cru à une température de $4^{\circ} \mathrm{C}$. Au moment où l'on expérimente, il y a donc deux facteurs qui interviennent, d'une part la pasteurisation dont l'action est fonction de l'intensité du traitement thermique, et d'autre part, le stockage du lait à basse température.

Cependant, comme nous l'avons montré dans des essais parallèles, l'allongement dû à la pasteurisation est plus important que celui dû au stockage et à peu près identique, que les essais de coagulation soient faits avant ou après conservation; nous ne tiendrons donc pas compte de cette dernière quand elle sera suivie de pasteurisation.

Il apparaît immédiatement que le temps de prise du lait pasteurisé, ainsi que le temps au bout duquel le caillé correspondant a acquis certaines propriétés, sont nettement plus importants que pour le lait cru. 
La différence est déjà nette en ce qui concerne le temps de prise puisque l'écart constaté entre 35 et $45^{\circ}$ n'est jamais inférieure à 1 minute.

Elle est encore plus nette pour le paramètre $k$ et atteint $2 \mathrm{mi}$ nutes, c'est-à-dire le double de l'écart précédent. Les propriétés mécaniques d'un coagulum provenant d'un lait pasteurisé sont donc obtenues après un temps significativement plus long que pour un lait cru, et cela d'autant plus que la température de pasteurisation est élevée. Ainsi, pour une température de $90^{\circ} \mathrm{C}$, nous avons obtenu des écarts allant de 4 à 7 minutes pour un temps de prise moyen en lait cru de $10 \mathrm{mn} 30 \mathrm{~s}$.

De plus, la pasteurisation raccourcit le palier au cours duquel le temps de prise ne varie pratiquement pas. Les courbes de la figure 5 montrent également que la valeur du paramètre $k$ est proportionnelle à celle de $r$. Or, par définition, $k$ correspond au temps au bout duquel le caillé a acquis des propriétés mécaniques bien définies que rien n'empêcherait de choisir identiques à celles d'un coagulum au moment du décaillage. On peut donc en conclure que le temps de coagulation varie proportionnellement au temps de prise, ce qui est conforme aux conclusions de Kelley, Swanson et Price [8].

5) Influence du stockage à basse température sur la coagulation [9], [10], [11], [12].

\section{a) Mode opératoire.}

Pour les mesures effectuées avant 'stockage, le lait est réparti en ballons de $200 \mathrm{ml}$ et conservé à une température voisine de $10^{\circ} \mathrm{C}$. Le temps écoulé entre la coagulation du premier échantillon et celle du dernier est de 2 h 30 environ. L'influence du stockage est étudiée sur une partie de ce lait conservé $24 \mathrm{~h}$ en chambre froide entre 0 et $4^{\circ} \mathrm{C}$. Dans le cas où les expériences sont faites sur lait cru et pasteurisé simultanément, les flacons de lait sont alternés de façon à rester dans des conditions expérimentales aussi voisines que possible.

\section{b) Résultats et discussion.}

Lait cru. - Une première série d'essais a donné pour $r$ et $k$, les valeurs figurant au tableau $\mathrm{V}$.

Ce tableau montre une augmentation sensible des temps de prise. Si l'on se place dans les conditions adoptées par Peltola et $\operatorname{Vogt}[9]$, cet accroissement n'atteint pourtant que 7 p. 100 contre 23 p. 100 pour ces auteurs. En revanche, l'augmentation du paramètre $k$ est moins sensible.

D'autres essais réalisés dans des conditions identiques, ont 
TABLEAU V

\begin{tabular}{|c|c|c|c|c|}
\hline $\begin{array}{l}\text { Tempé- } \\
\text { rature }\end{array}$ & $\begin{array}{c}r \\
\text { non stocké }\end{array}$ & $\begin{array}{c}r \\
\text { stocké }\end{array}$ & non $\stackrel{k}{s t o c k e ́}$ & $\begin{array}{c}k \\
\text { stocké }\end{array}$ \\
\hline $30^{\circ}$ & $9 \mathrm{mn}-9 \mathrm{~s}$ & $9 \mathrm{mn} 48 \mathrm{~s}$ & $6 \mathrm{mn} 45 \mathrm{~s}$ & $7 \mathrm{mn} 30 \mathrm{~s}$ \\
\hline $37^{\circ}$ & $7-$ & $7-33-$ & $3-48-$ & $4-03-$ \\
\hline $40^{\circ}$ & $6-36-$ & $7-18-$ & $3-54-$ & $4-$ \\
\hline $45^{\circ}$ & $6-36-$ & $7-$ & $4-06-$ & $4-06-$ \\
\hline $50^{\circ}$ & $12-$ & $12-06-$ & $\ldots$ & $\ldots$ \\
\hline
\end{tabular}

donné des résultats encore moins significatifs. On a même obtenu des temps de prise plus courts après stockage coïncidant avec des paramètres $k$ plus longs.

Lait pasteurisé. - Nous avons fait les expériences sur des laits pasteurisés à deux températures différentes : 75 et $90^{\circ} \mathrm{C}$. Le stockage ayant lieu après pasteurisation.

Dans le premier cas, les résultats observés ne sont pas significatifs. Les allongements de $r$ et de $k$ sont variables et demandent une étude plus approfondie. La figure 6 montre les deux thrombodynamogrammes obtenus à partir d'un lait traité à $75^{\circ}$, non stocké et stocké pendant $24 \mathrm{~h}$ à $1^{\circ} \mathrm{C}$. Si le lait conservé a coagulé plus d'une minute après le lait non stocké, le caillé correspondant a acquis les mêmes propriétés mécaniques que ce dernier, pratiquement dans les mêmes temps. De plus, les caractéristiques physiques maxima du coagulum sont sensiblement égales dans les deux cas.

Pour un lait pasteurisé à 90\%, l'effet du stockage est beaucoup plus important quelle que soit la température de l'expérience, comme le montre le tableau VI.

TABLEAU VI

\begin{tabular}{c|c|c}
\hline \hline Température & $\begin{array}{c}r \text { pasteurisé à } 90^{\circ} \\
\text { non stocké }\end{array}$ & $\begin{array}{c}r \text { pasteurisé à } 90^{\circ} \\
\text { stocké }\end{array}$ \\
\hline $30^{\circ}$ & $19 \mathrm{mn}$ & $24 \mathrm{mn} 30 \mathrm{~s}$ \\
$37^{\circ}$ & $13-30-$ & $15-30-$ \\
$40^{\circ}$ & $14-$ & $15-$ \\
$44^{\circ}$ & $16-45-$ & $19-$ \\
\hline \hline
\end{tabular}

A la température de coagulation de $30^{\circ}$, l'écart atteint plus de 20 p. 100. Nous n'avons pas mesuré le paramètre $k$ mais les différentes photographies de la figure 7 montrent que ses valeurs 


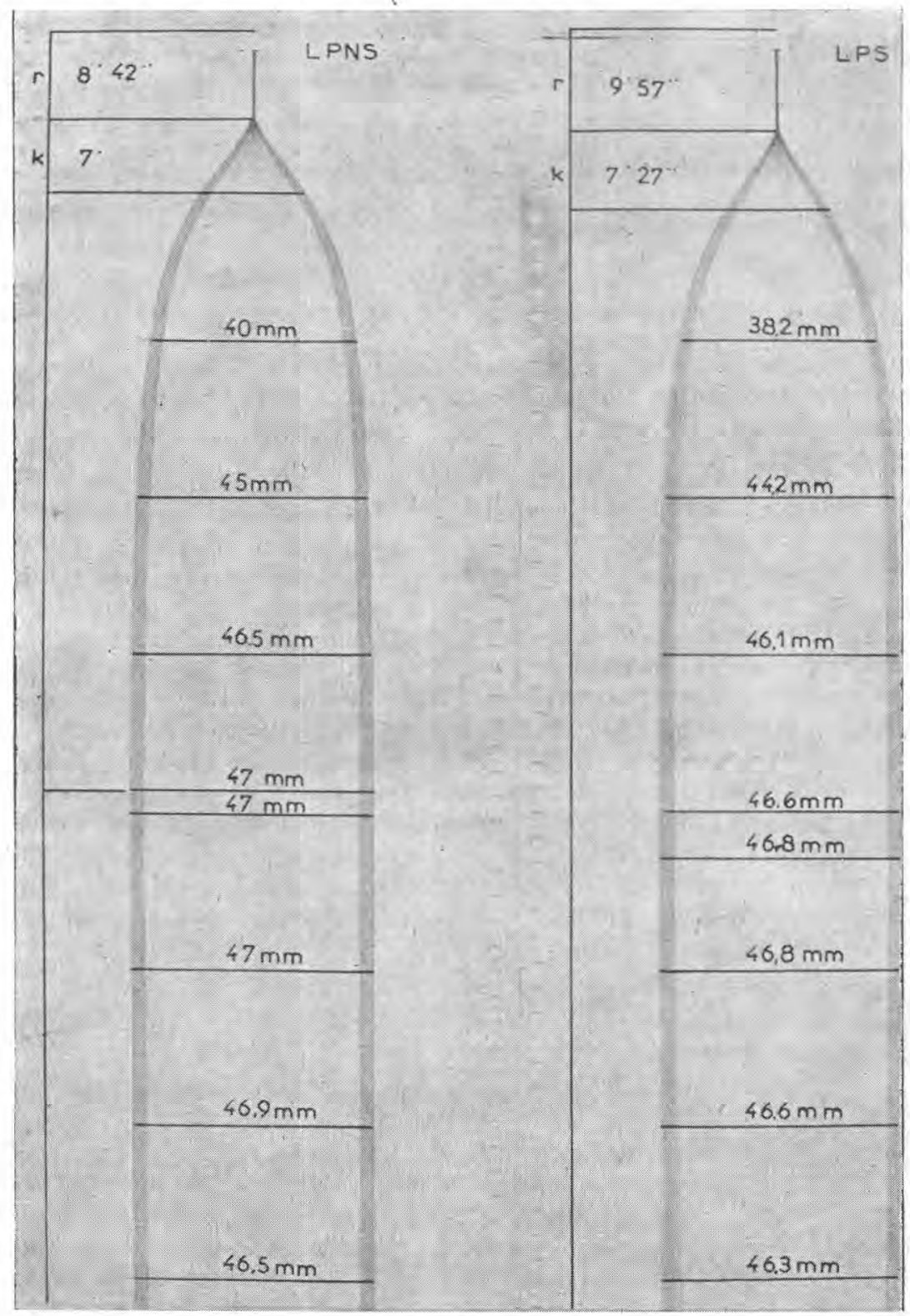

Eıg. 6. - Thrombodynamogrammes obtenus avant et après stockage pour un lait pasteurisé à $75^{\circ} \mathrm{C}$. 


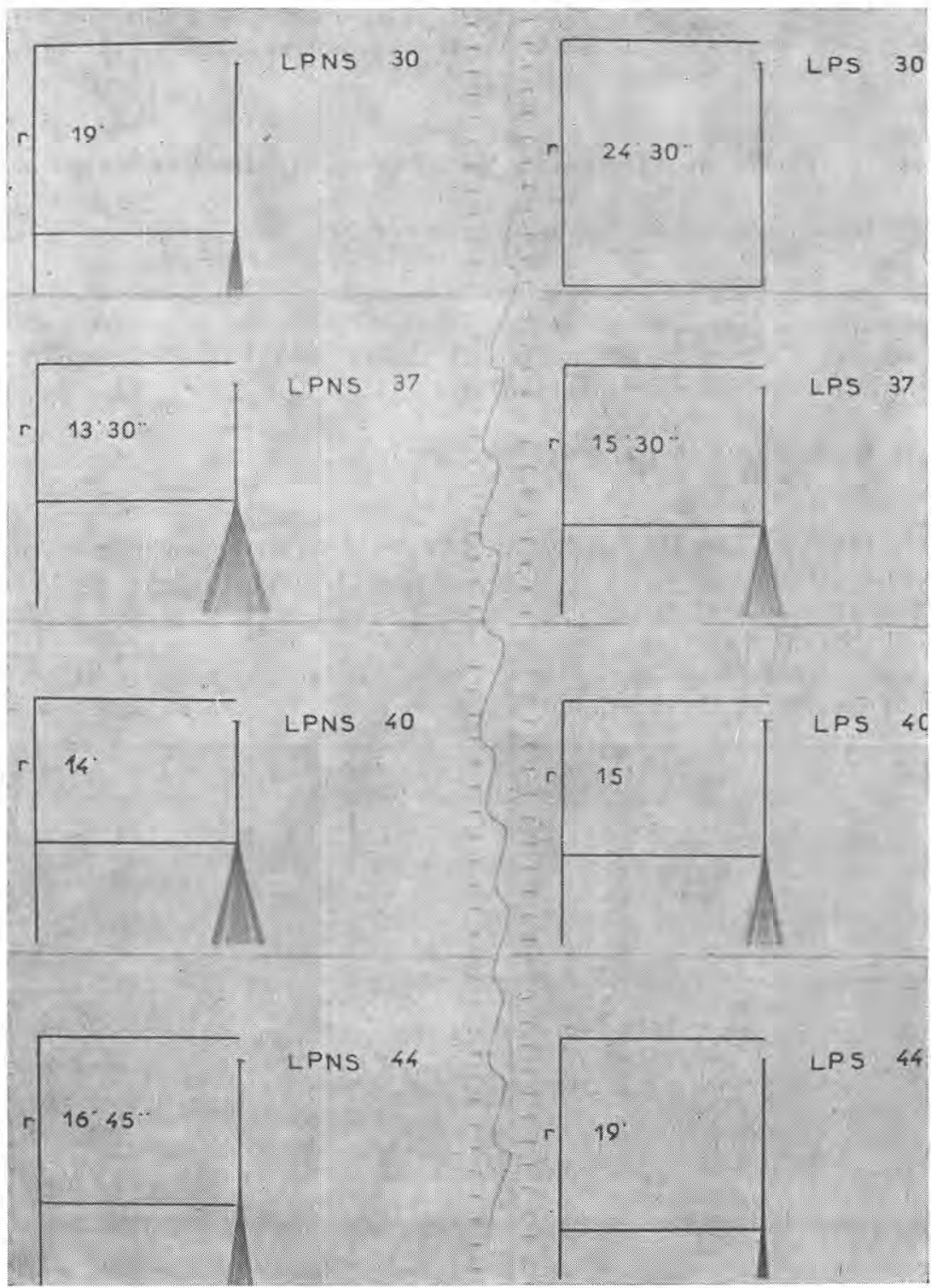

FIG. 7. - Action du stockage après pasteurisation à $90^{\circ} \mathrm{C}$.

auraient été plus importantes dans le cas du lait pasteurisé et stocké. Au bout du même temps, le caillé provenant du lait conservé est donc beaucoup plus mou que dans le cas du lait non stocké. Comme l'allongement du temps de prise après stockage entre 0 et $4^{\circ}$ paraît beaucoup moins net et constant dans le cas 
de lait cru ou pasteurisé à basse température, son absence est peut-être explicable dans ces cas par une intervention diastasique qui provoquerait un début de protéolyse.

\section{II. - Etude de l'influence de la concentration en calcium}

Dans un premier temps, nous avons étudié l'influence de la teneur en calcium sur les paramètres $r$ et $k$, en prenant comme substrat soit du lait cru, soit du lait reconstitué. Nous avons ensuite examiné ce que devenaient les propriétés mécaniques maxima du coagulum lorsqu'on augmentait la teneur en calcium en prenant du lait cru comme substrat [13], [14], [15].

\section{A) Evolution des paramètres $r$ et $k$.}

1) Cas du lait cru.

Les essais ont été faits sur du lait cru dont la teneur en calcium était inítialement égale à $1,05 \mathrm{~g}$ par litre. L'emprésurage a été fait à $36^{\circ} \mathrm{C}$ par $1 \mathrm{ml}$ de présure Hauser de force 1000 pour $100 \mathrm{ml}$ de lait. La figure 8 reproduit les thrombodynamogrammes obtenus. Les résultats numériques sont consignés dans le tableau VII et ont permis de tracer la courbe $c$ de la figure 9 .

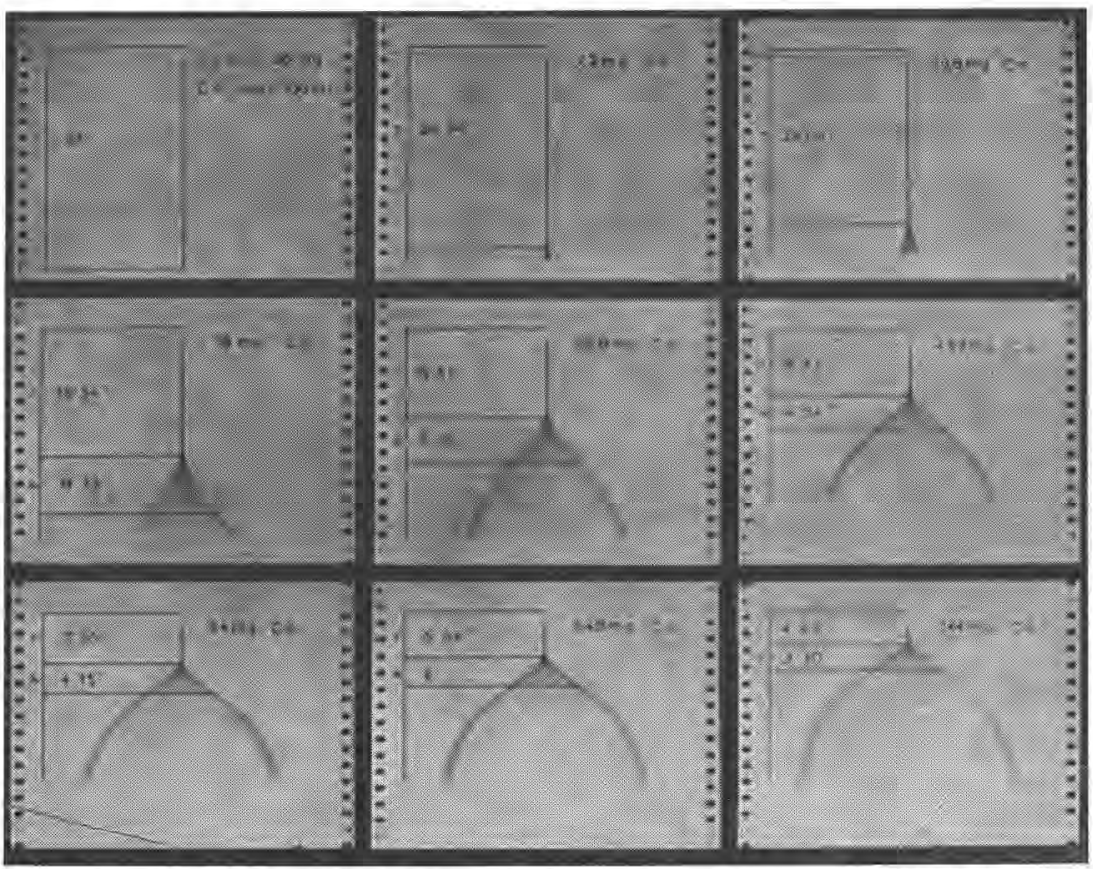

Fig. 8, - Influence de la concentration en caleium. Cas du lait cru. 
TABLEAU VII

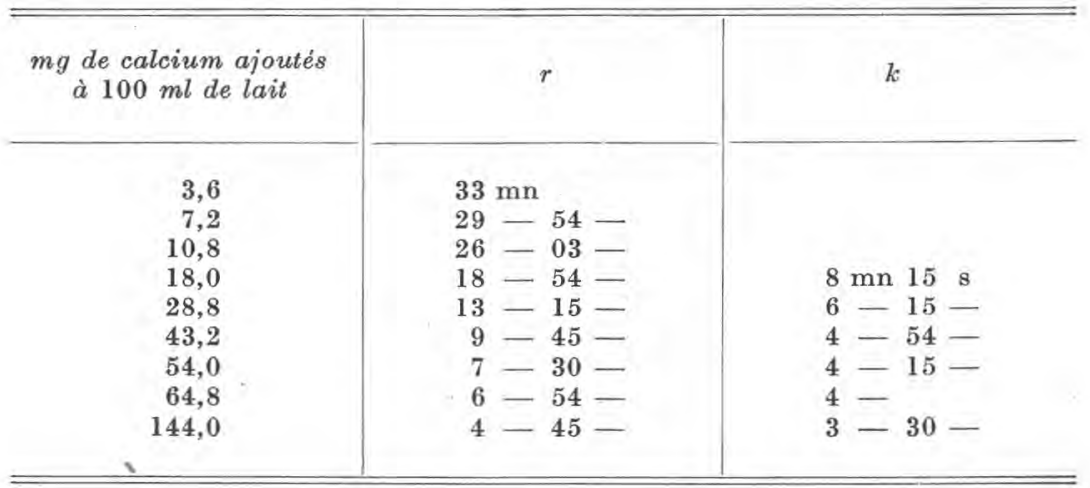

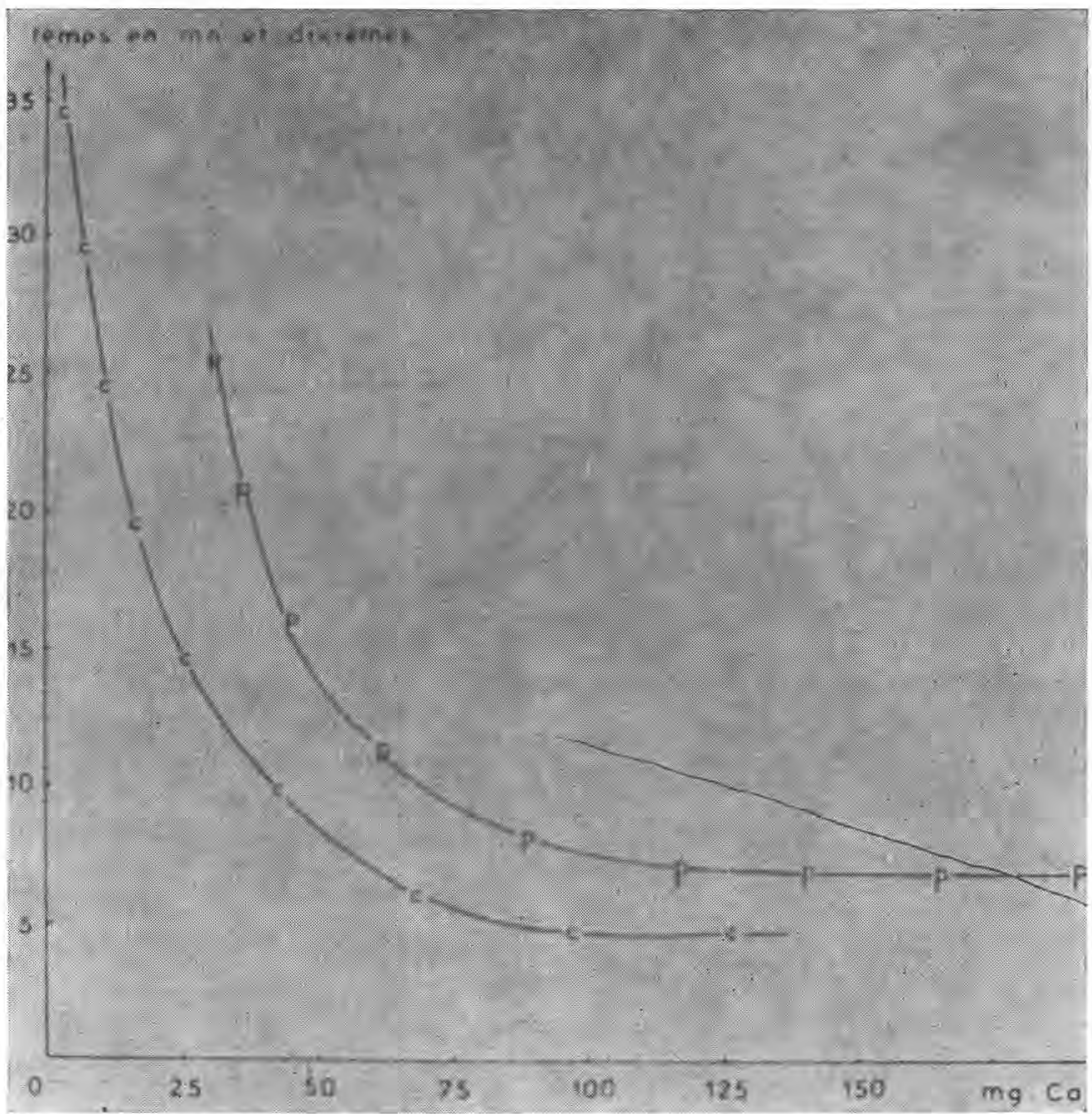

Fig. 9. - Evolution des temps de prise en fonction de la teneur en calcium. $p=$ cas du lait reconstitué, 
L'augmentation de Ia teneur en calcium du lait entraîne d'abord une diminution très rapide $d u$ temps de prise et une plus prompte acquisition du caractère "fermeté du caillé » jusqu'à une addition d'environ $90 \mathrm{mg}$ de calcium pour $100 \mathrm{ml}$ de lait. Puis, cette action se ralentit et on n'observe plus qu'une évolution très légère des paramètres $r$ et $k$ alors que la dose de calcium s'accroît considérablement.

2) Cas du lait reconstitué (fig. 10).

Les résultats sont identiques à ceux du cas précédent ainsi que le montre la courbe $p$ de la figure 9 . Il faut remarquer que, pour un même résultat, la quantité de calcium nécessaire est beaucoup plus importante dans le cas du lait reconstitué que dans le cas du lait cru, ce qui est normal étant donné l'action de la température sur le calcium du lait au cours de la fabrication de la poudre.

\section{B) Evolution du paramètre Am.}

Ce paramètre représente les propriétés mécaniques maxima que peut fournir un coagulum dans des conditions bien déterminées ; si l'on veut préciser encore cette nouvelle variable, il faut lui adjoindre une notion de temps et donner les résultats sous la forme : les propriétés mécaniques du caillé ont atteint un maximum Am au bout d'un temps S. Cela permettra une comparaison plus facile des divers coagulums obtenus.

Pour étudier ces variations, nous sommes partis d'un lait cru, formolé à raison d'une partie pour 2000 , additionné d'une quantité variable de calcium et emprésuré à $35^{\circ} 5$ par $1 \mathrm{ml}$ de présure Hauser de force 1000 . Les résultats obtenus sont ceux du tableau VIII.

L'examen de ces données montre que l'action du calcium dans les propriétés mécaniques du caillé est double. D'une part, il

TABLEAU VIII

\begin{tabular}{|c|c|c|c|c|}
\hline $\begin{array}{c}\text { Calcium } \\
\mathrm{mg} / 100 \mathrm{ml}\end{array}$ & $r$ & $k$ & $A m(m m)$ & $S$ \\
\hline $\begin{array}{c}0 \\
10,80 \\
21,60 \\
32,40 \\
43,20 \\
54,00 \\
64,80 \\
86,40\end{array}$ & $\begin{array}{l}9 \mathrm{mn} 15 \mathrm{~s} \\
5-51- \\
2-51- \\
2-12- \\
1-54- \\
1-48- \\
1-21- \\
1-12-\end{array}$ & $\begin{array}{l}7 \mathrm{mn} \\
3- \\
2- \\
1-33- \\
1-21- \\
1-15- \\
1-12- \\
1-12-\end{array}$ & $\begin{array}{l}49 \\
58,4 \\
60 \\
63,6 \\
66,4 \\
66,4 \\
68,0 \\
68,0\end{array}$ & $\begin{array}{r}107 \mathrm{mn} \\
77- \\
73- \\
47- \\
98- \\
106- \\
100- \\
95-\end{array}$ \\
\hline
\end{tabular}




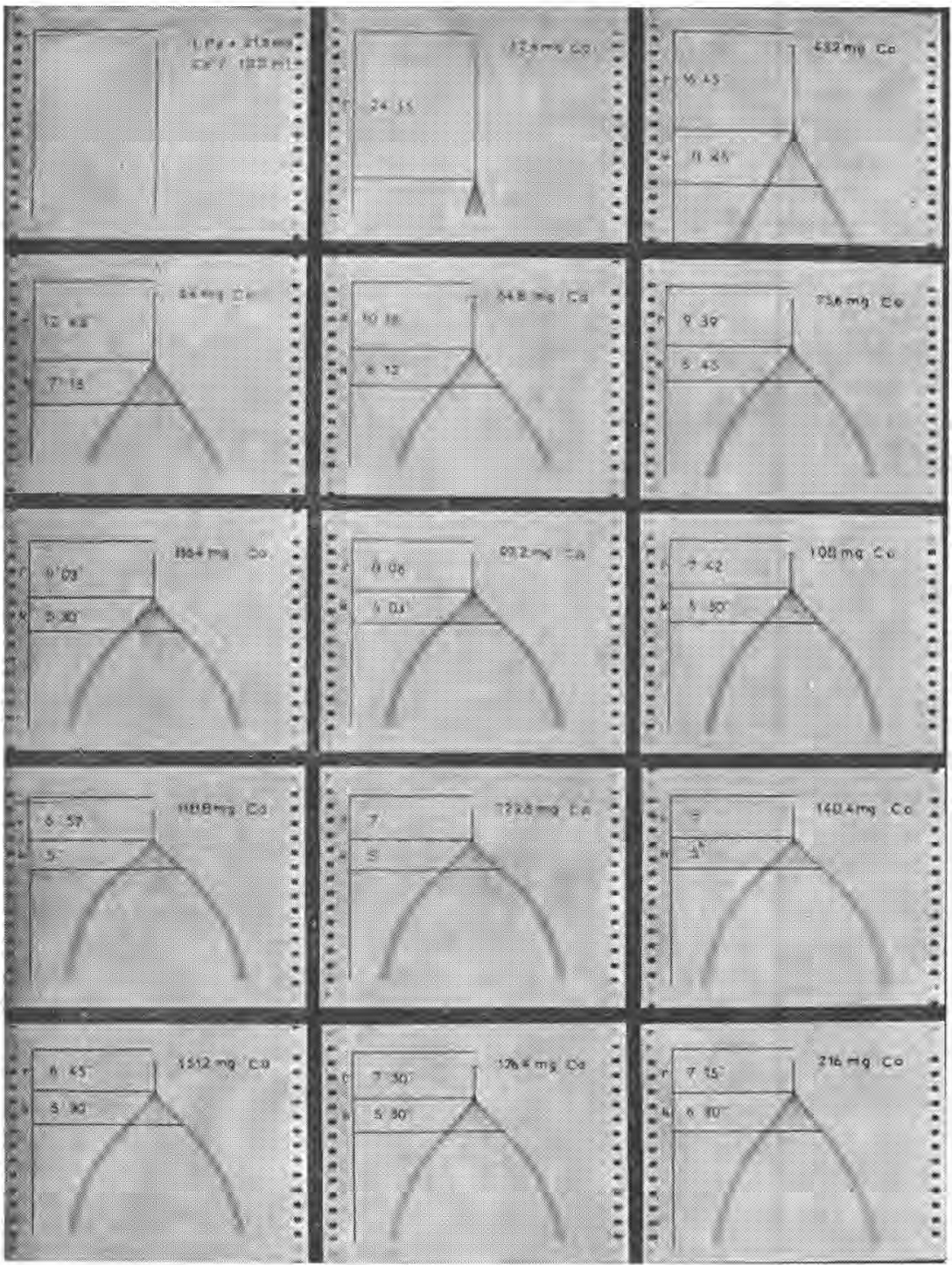

Fia. 10. - Influence de la concentration en calcium. Cas du lait reconstitué.

permet d'obtenir une certaine fermeté en un temps d'autant plus court que sa concentration est élevée, d'autre part, il augmente la valeur absolue de ce caractère. Ces deux actions sont évidemment limitées; dans le cas évoqué, la limite se situe aux environs de $43,2 \mathrm{mg}$ de calcium pour $100 \mathrm{ml}$ de lait. Au-delà de cette concen- 
tration, les temps nécessaires pour obtenir Am sont sensiblement identiques, de même que la valeur de ce paramètre.

L'action de la teneur en calcium ne doit pas être confondue avec celle de l'extrait sec. En effet, déjà à elle seule, une augmentation de l'extrait sec entraîne une diminution des paramètres $r$ et $k$ et un accroissement de fermeté, comme on peut le montrer simplement à partir de poudre de lait. Pour cela, il suffit de préparer des laits reconstitués avec des quantités croissantes de poudre. Chacun renfermera le même pourcentage de calcium par rapport à l'extrait sec qui, par contre, sera variable. Nous avons fait un essai dans ce but en emprésurant avec $1 \mathrm{ml}$ de présure de force 1000 pour $100 \mathrm{ml}$ de lait, à une température de $35^{\circ} 5$. Les résultats obtenus sont ceux du tableau IX.

TABLEAU IX

\begin{tabular}{|c|c|c|}
\hline Lait reconstitué à ' & $r$ & $k$ \\
\hline $\begin{array}{l}10 \% \\
12,5 \% \\
15 \% \\
17,5 \%\end{array}$ & $\begin{array}{l}16,45 \\
13,15 \\
12,45 \\
12,09\end{array}$ & $\begin{array}{r}18,54 \\
7,30 \\
4,27 \\
3,09\end{array}$ \\
\hline
\end{tabular}

La réduction des temps de prise est beaucoup moins forte dans ces conditions qu'en faisant varier la dose de calcium. En revanche, le coagulum acquiert tout aussi rapidement ses propriétés mécaniques comme le montre la figure 11 .

Les actions du calcium et de l'extrait sec sont donc apparemment semblables pendant le phénomène de la coagulation par la présure en ce qui concerne les temps de prise et le caractère "fermeté " du caillé.

\section{III. - Conclusion}

L'appareil utilisé nous a permis de suivre l'action de la température sur la coagulation d'une façon suffisamment sensible puisque des variations de température de $2^{\circ} \mathrm{C}$ seulement ont entraîné des différences significatives pour les valeurs des paramètres $r$ et $k$. De même, l'influence de la pasteurisation est très nette et ce d'autant plus que la température du traitement est élevée, alors que l'action du stockage à basse température est beaucoup plus discutable et demande une étude plus approfondie. Enfin, les différences provoquées entre les coagulations de laits identiques par la seule variation de la teneur en calcium montrent bien la place importante occupée par cet élément en fromagerie. 


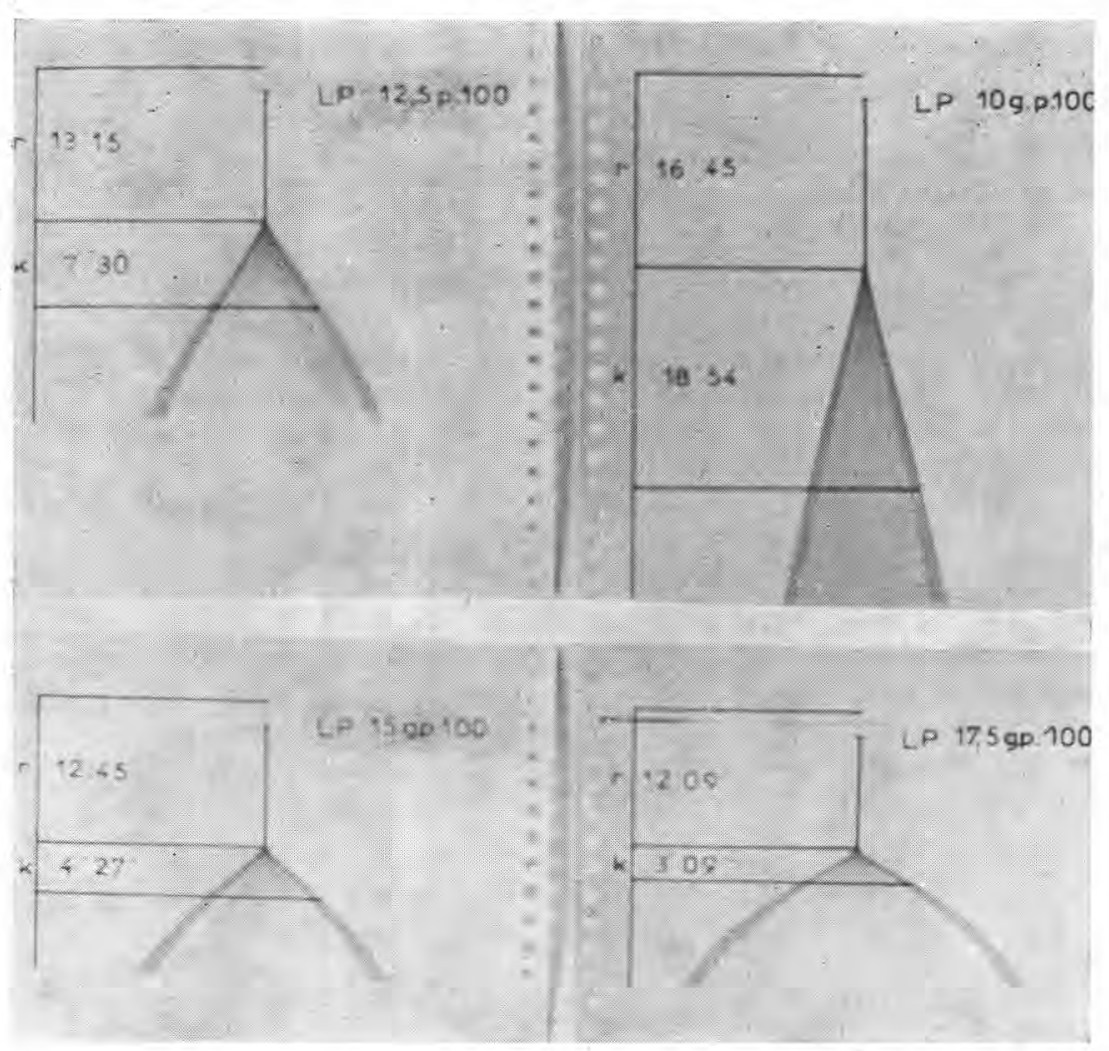

Fig, 11 - Influence de la concentration en extrait sec.

D'un autre côté, l'appréciation par le fromager de l'état physique du caillé est indispensable à différents stades d'une fabrication, car les qualités du produit fini vont en dépendre. C'est ainsi que, dans certains cas, le moment du décaillage est primordial car il conditionne l'égouttage. Aussi a-t-on cherché depuis longtemps à mesurer les temps de prise et à apprécier les propriétés mécaniques du caillé [5], [17], [18], [19], de façon à réduire au minimum les risques d'accidents consécutifs à une erreur d'appréciation toujours possible.

Dans l'état actuel des essais, le thrombélastographe de Hartert, dont la sensibilité a été fortement accrue, permet de mesurer les temps de prise et les propriétés mécaniques d'un coagulum d'une manière suffisamment précise et reproductible. Il semble donc qu'il puisse jouer un rôle en pratique dans la conduite des fabrications ou dans la prévision de leur déroulement. Il est en effet possible de comparer entre eux les thrombélastogrammes donnés par des 
laits différents et, grâce à l'expérience et aux résultats ainsi acquis, on pourra peut-être intervenir sur les facteurs de la coagulation pour obtenir un thrombélastogramme voisin d'un thrombélastogramme correspondant à la coagulation optimum pour une fabrication déterminée.

\section{Résumé}

Le thrombélastographe de Hartert, appareil sensible, précis et fidèle, a permis d'étudier l'action de la température, de l'extrait sec et du calcium sur la coagulation par la présure, de différents laits reconstitués, crus ou pasteurisés.

\section{Summary}

Thanks to Hartert's thrombelastograph, a sensitive, precise and reliable apparatus, it has been possible to study the effect of the temperature, the dry matter and the calcium on rennet coagulation of reconstituted, raw or pasteuriszed milks.

\section{BIBLIOGRAPHIE}

[1] G. Marchal, M. E. Leroux, M. Samana. - Atlas de Thrombodynamographie. Service de Propagande. 1962. Edition Information, 14, rue Drouot, Paris $\left(9^{\mathrm{e}}\right)$.

[2] J. JACQUET, H. MARCAIS. - Enregistrement graphique du phénomène de coagulation du lait. C. R. Ac. Agric., 1964, 50, pp. 1272-1280.

[3] H. Marcais. - Emploi de la Thrombélastographie pour l'étude de la coagulation du lait. Le Lait, 1965, 45, no 445-446, p. 241.

[4] F. Frentz. - Application de la thrombélastographie de Hartert à l'étude de la coagulation du lait. Le Lait, 1965, 45, no 448, pp. 489508.

[5] J. M. MAN (DE), S. C. Batra. - Measurement of the rennet clotting time of milk with an automatic clot-timer. Dairy Industries, January 1964, p. 32 .

[6] A. Kannan, R. Jenness. - Relation of milk serum proteins and milk salts to the effects of heat treatment on rennet clotting. J. Dairy Sci., 1961, 44, no 5, p. 808.

[7] G. M. Morr. - The effect of heat upon the rennin coagulation. J. Dairy Res. 1930 , 2, p. $68 ; 3$, p. 80 .

[8] L. A. Kelley, A. M. Swanson, W. V. Price. - Studies relating to rennet coagulation time and rennet curd tension. J. Dairy Sci., Abstracts, 1951, p. 483.

[9] E. Peltola, P. Vogt. - The effect of cold ageing on the rennetting of milk. XVe Congrès intern. Lait., 1959, 1, p. 268.

[10] C. Alars. - Science du lait. Principes des techniques laitières Edit. Sep., Paris (1er), 1965, p. 518. 
[11] A. Monvoisin. - La conservation par le froid des denrées périssables. Dunod, 1950, Paris.

[12] R. Coppini, A. Morozzr. - Recherches physiques, chimiques et bactériologiques sur le lait congelé. Industrie lait., 1959, no 148, p. 19.

[13] A. M. Guerault. - Quelques réflexions d'ordre pratique sur le rôle du calcium en fromagerie. Le Lait, 1957, no 369 , p. 593.

[14] M. Hilgeman, R. Jennes. - Observations on the effect of heat treatement upon the dissolved calcium and phosphorus in skimmilk. Cité par J. Dairy Sci., no 6, juin 1951.

[15] H. Tessier, Dyson Rose. - Calcium ion concentration in milk. J.Dairy Sci., 41, p. 351.

[16] C. Alats. - Science du lait. Principes des techniques laitières. Edit. Sep., Paris (1er), 1965, p. 520.

[17] H. Roeder. - Méthode d'observation de la coagulation du lait, Molkerei Zeit., LII, 1938, no 87, p. 2637. Cité par Le Lait, 1944, p. 279.

[18] J. Burnett, G. W. Scott Blair. - A speed-compensated torsiometer for measuring the setting of milk by rennet. Dairy Industries, march 1963.

[19] J. Burnets, G. W. Scott Blair. - Note on the torsiometer for measuring setting of milk by rennet. Dairy Industries, February 1964. 